\title{
Comparison of Electrical Cardiometry and Transoesophageal Doppler for Haemodynamic Monitoring During Living Donor Liver Transplantation.
}

Khaled A. Yassen ${ }^{1}$, Nagwa Ibrahem Mowafy ${ }^{1}$, Eman Sayed Ibrahem ${ }^{1}$, Nagwa Mohamed Doha ${ }^{2}$, Ashraf Mohamed Moustafa ${ }^{2}$ Anaesthesia Departments of Liver Institute ${ }^{1}$ and Faculty of Medicine ${ }^{2}$ Menoufia University, Shebeen Elkom City, Egypt

\section{Background and Aim of the Work}

Left ventricle cardiac output can be calculated non-invasively with Electrical Cardiometry $(E C)$ utilizing the thoracic electrical bioimpedance or minimal invasively with Transesophageal Doppler (TED) measuring descending aorta blood flow.

The aim of the work is to compare EC to TED regarding cardiac output (CO) and guided fluid administration, as well as reporting haemodynamic changes during transplantation.

\section{Patients and Methods}

First of all ethics committee approval, Pan African Clinical Trial Registry (PACTR201701001990415) and informed consent for this prospective hospital based comparative study was obtained. 47 adults ( 3 excluded): EC gp, $(n=22)$ and TED gp ( $n=22)$ Following anaesthesia, TED probe (CardioQ, Deltex, Chichester, UK) was passed orally into mid-esophagus. Cardiometry skin sensors were applied simultaneously (ICON, Osypka Medical, Germany).In EC gp Anaethesist were blinded to TED and vice versa. Ringer's acetate infused at $6 \mathrm{~m} \mathrm{l} \mathrm{kg} / \mathrm{h}$. Albumin $5 \% 3 \mathrm{ml} / \mathrm{kg}$ infused when stroke volume variation (SVV) (\%) in EC > 10\% or corrected flow time $(\mathrm{FTc})(\mathrm{mSec})$ in TED $<350 \mathrm{msec}$. Blood products were guided by rotational thromboelastometry.

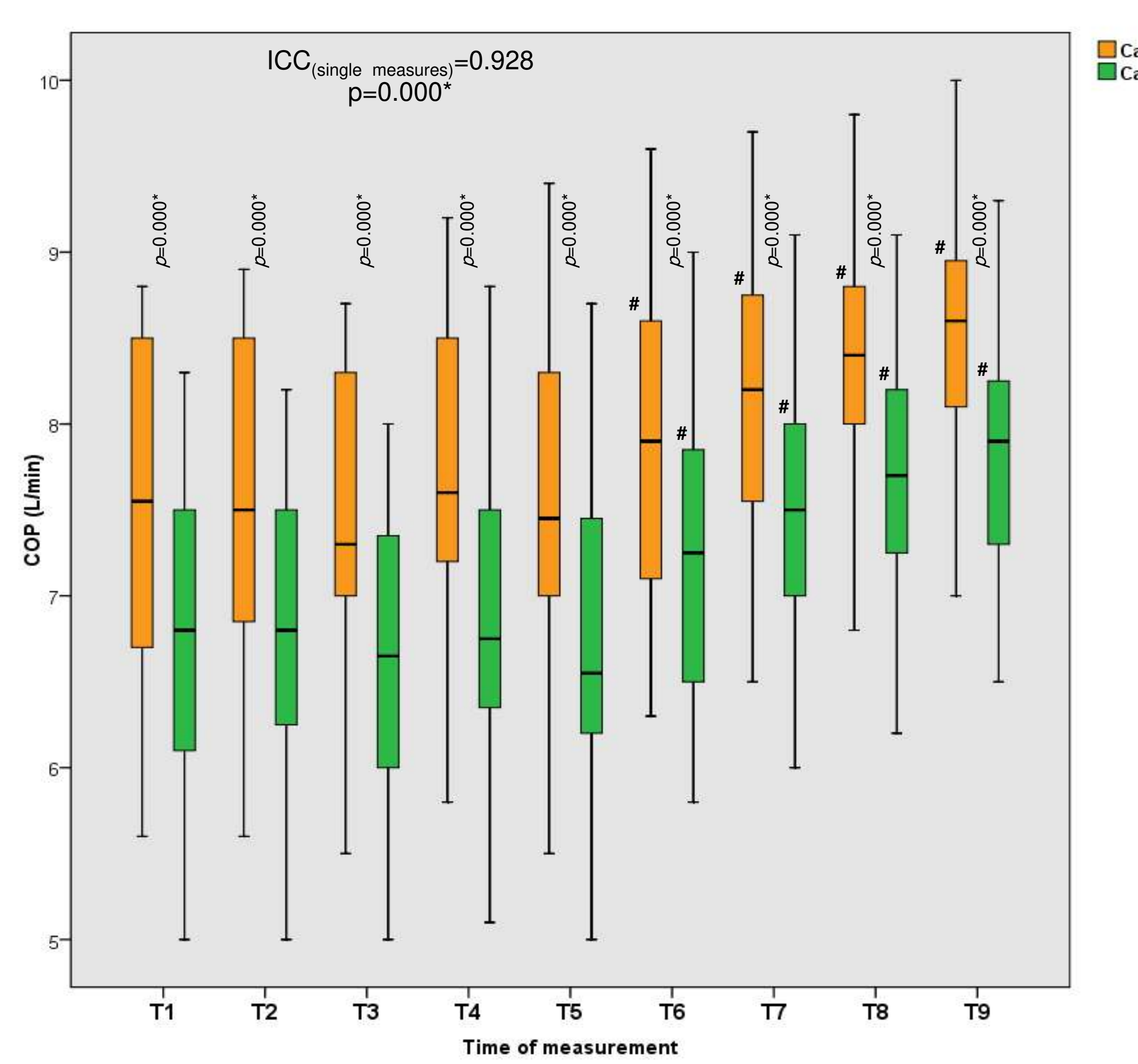

\# significant difference comp
ICC: Intraclass correlation

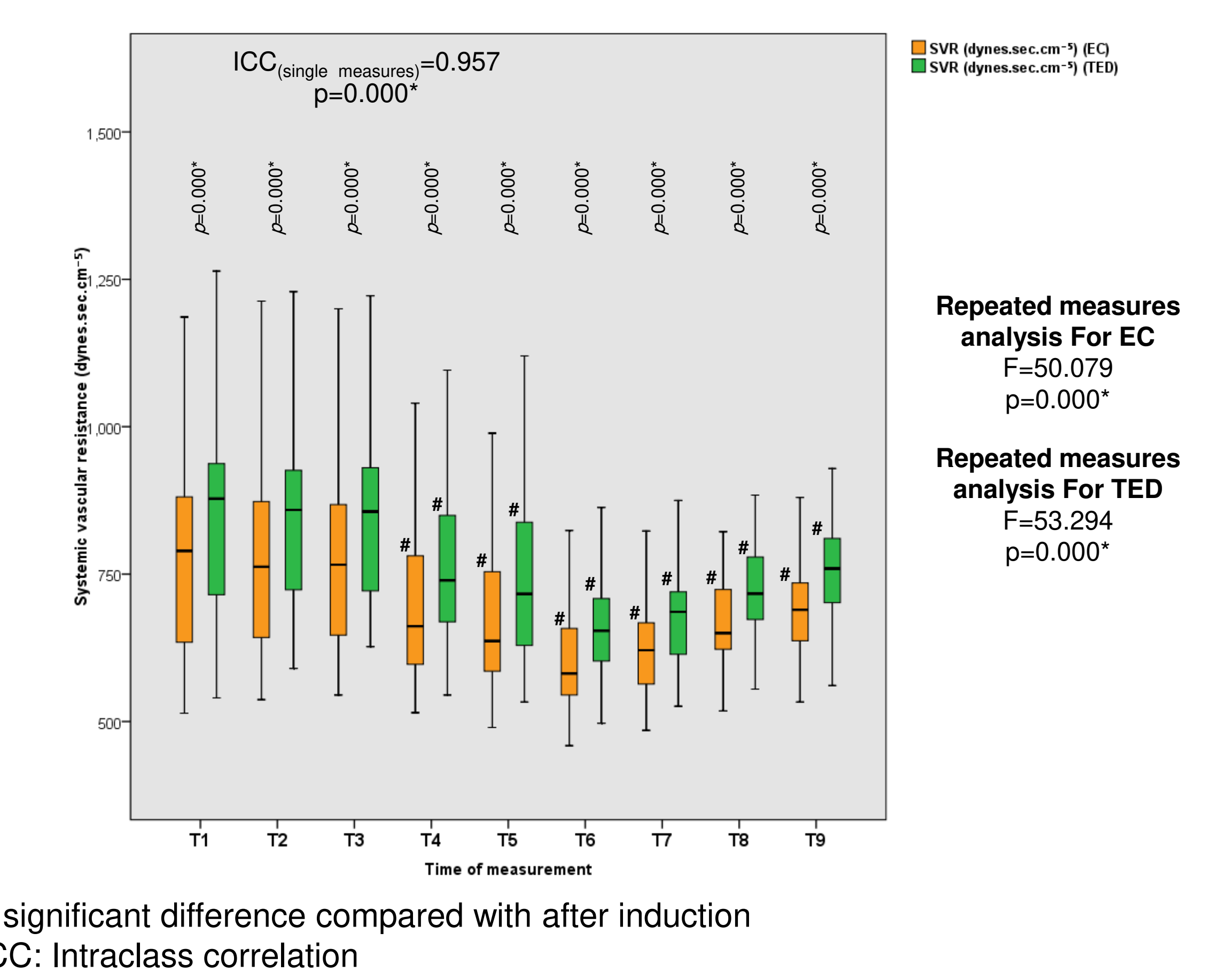

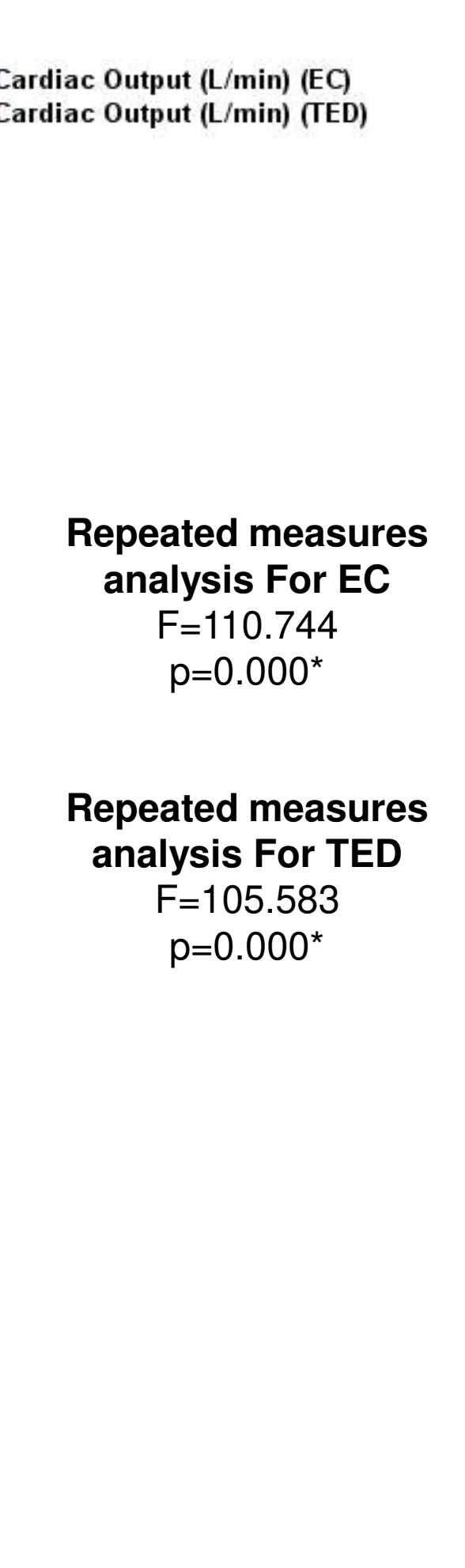
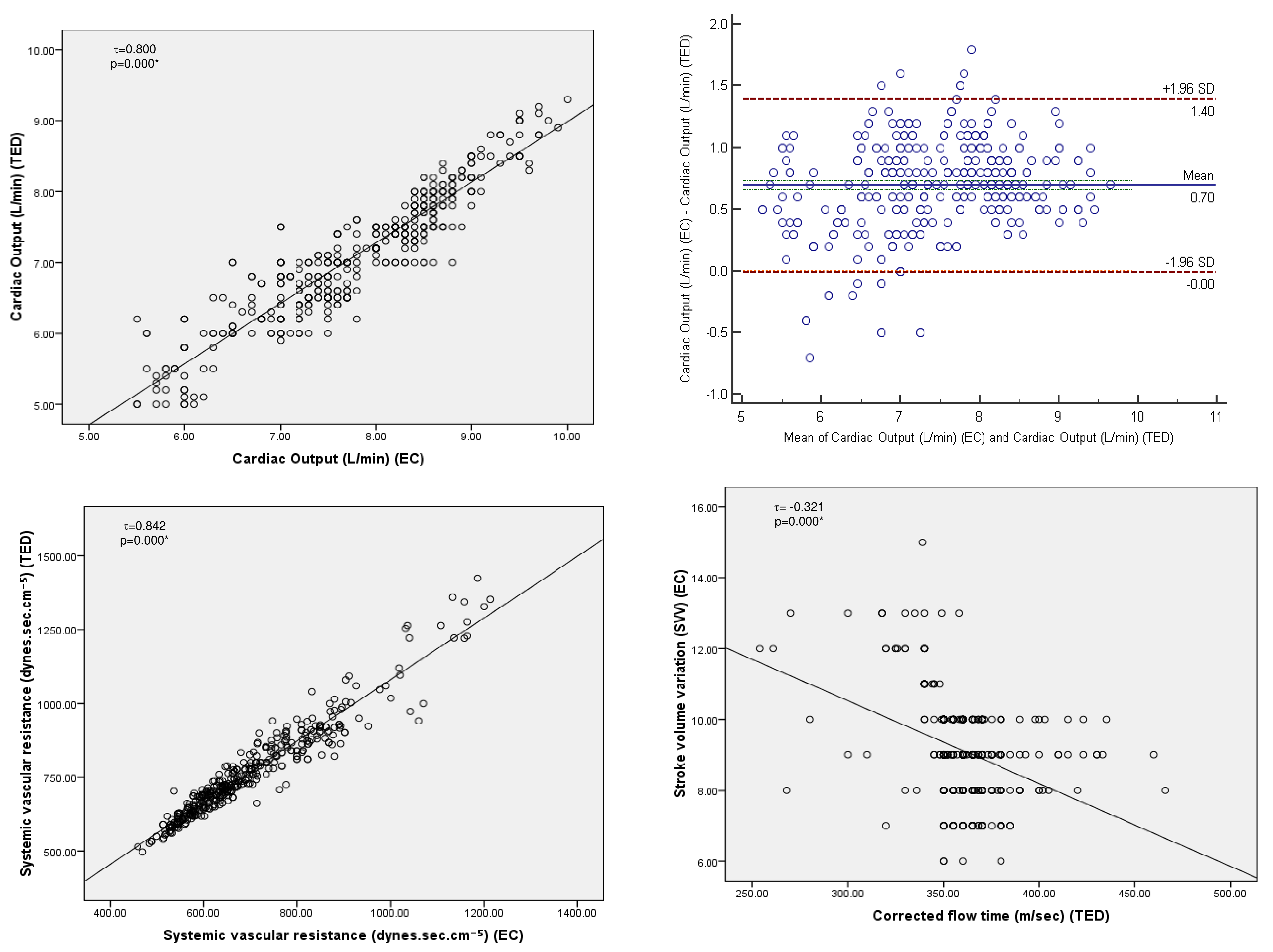

\begin{tabular}{ccc}
$\begin{array}{c}\text { EC (Cardiometry) } \\
(\mathrm{n}=22)\end{array}$ & $\begin{array}{c}\text { TED (Cardio-Q) } \\
(\mathrm{n}=22)\end{array}$ & $\begin{array}{c}\text { Test of significance } \\
p(\text { value })\end{array}$ \\
\hline $800(600-1000)$ & $850(800-1000)$ & $\mathrm{Z}_{(\mathrm{MW})}=1.269$ \\
& & $p=0.204 \mathrm{NS}$ \\
$5500(5200-6000)$ & $5525(5200-6000)$ & $\mathrm{Z}_{(\mathrm{MW})}=0.241$ \\
& & $p=0.810 \mathrm{NS}$
\end{tabular}

\section{Conclusion}

- EC \& TED can be used interchangeably as cardiac output, haemodynamic monitoring \& goal directed fluid management.

- TED CO was lower than EC CO despite reporting similar changes during different phases of LT

- Both techniques were able to demonstrate significant haemodynamic changes specially with reperfusion 